\section{Avaliação dos principais efeitos endócrinos tardios em crianças e adolescentes sobreviventes ao tratamento de neoplasias malignas}

\author{
Evaluation of the main late endocrine effects in children \\ and adolescents after treatment of malignancies
}

Hilton Kuperman', Claudilene Battistin', Ana Cristina Fraga Moreira', Ana Lucia Cornacchioni ${ }^{2}$,Vicente Odone Filho ${ }^{2}$,Nuvarte Setian', Durval Damiani'

\section{RESUMO}

Objetivo: Relatar os efeitos endócrinos tardios em crianças e adolescentes após tratamento oncológico e associá-los à doença de base e ao tratamento. Sujeitos e métodos: Foram realizadas avaliações clínicas e laboratoriais visando à detecção de distúrbios endócrinos em 320 pacientes após terapia oncológica, seguidos por seis anos. Resultados: Em 94 pacientes, detectaram-se: 32 pacientes apresentaram baixa estatura (nove em tratamento com hormônio de crescimento), 14 tiveram puberdade precoce (10 em uso de análogo de $\mathrm{GnRH}$ ) e 19 revelaram ser portadores de distúrbios de tireoide (12 com hipotireoidismo, seis com nódulos de tireoide e um com tireoidite linfocitária crônica). Obesidade foi encontrada em 18 deles. Seis com diabetes insípido e cinco com puberdade atrasada, três com pan-hipopituitarismo. Houve associação entre a radioterapia e a presença de endocrinopatias. Conclusão: Noventa e quatro de $320(30 \%)$ dos pacientes fora de terapia apresentaram alteração endócrina, o que enfatiza a importância do seguimento precoce e regular, possibilitando-lhes, com tratamento, melhor qualidade de vida. Arq Bras Endocrinol Metab. 2010;54(9):819-25

Descritores

Neoplasias; crianças; adolescentes; endocrinopatias; sobreviventes de câncer

\begin{abstract}
Objective: To report the main endocrine effects after cancer treatment in children and adolescents and associate them to the disease and its treatment. Subjects and methods: Clinical and lab evaluation for endocrinopathy was performed in 320 patients after cancer therapy have been followed for six years. Results: The most prevalent endocrine late effects in patients were: 32 patients had short stature, nine of them were under growth hormone therapy. Precocious puberty was found in 14 patients, 10 of them received $\mathrm{GnRH}$ analog. Thyroid diseases were present in 19 patients ( 12 with hypothyroidism; six with thyroid nodules/cysts; one with chronic lymphocytic thyroidytis). Obesity was found in 18 patients. Six presented insipidus diabetes, five delayed puberty and three panhypopituitarism. Radiation was associated with the appearance of the aforementioned endocrinopathies. Conclusion: Ninety four of $320(30 \%)$ patients presented endocrine late effects which emphasize the importance for these patients to be regularly followed-up in order to precociously diagnose endocrine late effects and provide them a better quality of life. Arq Bras Endocrinol Metab. 2010;54(9):819-25
\end{abstract}

Keywords

Neoplasms; child; adolescent; endocrine diseases; cancer survivors
Unidade de Endocrinologia Pediátrica, Instituto da Criança, Hospital das Clínicas, Faculdade de Medicina da Universidade de São Paulo (ICr-HC-FMUSP), São Paulo, SP, Brasil

2 Unidade de Oncologia Pediátrica, ICr-HC-FMUSP, São Paulo, SP, Brasil
Correspondência para:

Hilton Kuperman

Rua Conselheiro Brotero, 1182, ap. 182

01232-010 - São Paulo, SP, Brasil hkuperman@terra.com.br

Recebido em 21/Set/2009 Aceito em 4/Out/2010 


\section{INTRODUÇÃO}

$\mathrm{O}^{2}$ avanço na padronização de protocolos de quimioterapia, cirurgia e radioterapia para o tratamento do câncer infantil resultou em maior sobrevida dos pacientes oncológicos. Sabe-se que a taxa atual de sobrevivência é de cerca de $80 \%$ nas leucemias e $90 \%$ na doença de Hodgkin $(1,2)$. Concomitantemente, houve um aumento das complicações decorrentes da terapia prévia, entre as quais os distúrbios endócrinos correspondem a $20 \%$ a $50 \%$ dos casos (1).

Os efeitos tardios podem decorrer não apenas da condição clínica e da predisposição genética do paciente, mas, principalmente, do tipo de tratamento ao qual foi submetido. O tipo e a dose dos agentes quimioterápicos, além da intensidade e região corpórea de irradiação, são fatores que podem ser relacionados às manifestações clínicas tardias dos pacientes (3).

Nos indivíduos que receberam radioterapia, especialmente de crânio e eixo, o risco de acometimento é aumentado, o que pode tornar vulnerável a região hipotálamo-hipofisária, levando a uma secreção deficiente de hormônio de crescimento, além de alterações da puberdade tanto precoce como tardia (3-8).

A incidência de síndrome metabólica e, sobretudo, de obesidade também é crescente $(6,9,10)$. Pacientes do sexo feminino e os sobreviventes de leucemia linfocítica aguda (LLA) apresentam maior suscetibilidade $(9,10,11)$. A irradiação de crânio e os tumores que acometem a região hipotálamo-hipofisária parecem ser um dos principais fatores etiológicos $(3,9)$. Assim, doenças cardiovasculares e dislipidemias assumem papel importante no enfoque de intervenções preventivas e terapêuticas logo nos primeiros anos de seguimento.

A radioterapia, notadamente na região cervical, pode levar a um aumento na incidência de tireoidopatias, nódulos e tumores $(6-8,12,13)$.

Os efeitos endócrinos tardios são multifatoriais e requerem um seguimento de longo prazo, sendo necessária uma análise constante.

O presente estudo objetiva relatar os efeitos endócrinos tardios em crianças e adolescentes após tratamento oncológico e associá-los à doença de base e ao tratamento.

\section{CASUÍSTICA E MÉTODOS}

A partir de 2002, foi instituído o grupo de pacientes Fora de Terapia no Instituto de Tratamento do Câncer
Infantil (Itaci), do Instituto da Criança do Hospital das Clínicas da Faculdade de Medicina da Universidade de São Paulo (ICr-HC-FMUSP). Trezentos e vinte dos que sobreviveram ao tratamento de neoplasias - que constituiu em cirurgia, quimioterapia e radioterapia, conforme o protocolo adotado para a doença específica - foram acompanhados pelo grupo de Fora de Terapia do Itaci. O seguimento envolveu o acompanhamento de efeitos tardios. Os pacientes foram encaminhados para avaliação endócrina inicialmente cinco anos após o término da terapia, sendo esse prazo posteriormente reduzido para dois anos ou quando houvesse necessidade dessa avaliação pelo grupo de Fora de Terapia.

Esses 320 pacientes foram avaliados no ambulatório de endocrinologia pediátrica do Itaci no período de setembro de 2002 a agosto de 2008. Esse grupo constitui a nossa amostra de estudo. Foram aferidos dados antropométricos, como altura, peso e índice de massa corpórea (IMC), sendo usados como referência as tabelas do NCHS-2000 para avaliação dos percentis e de seu índice $\mathbf{z}$ quando apropriado e o estádio puberal segundo os critérios de Tanner. Além disso, os pacientes foram avaliados laboratorialmente e com exames de imagem, conforme indicação clínica e diagnóstica, e também realizaram-se exames de rotina em todos eles, que incluíram: hemograma, dosagem sérica de eletrólitos, funções hepática e renal e avaliação da idade óssea (método Greulich-Pyle).

Para avaliação da deficiência de hormônio de crescimento $(\mathrm{GH})$, foram realizados: dosagens de IGF-1 e IGFBP-3 séricos (imunoquimioluminométrico), testes de estimulação hormonal (clonidina e/ou glucagon) para avaliação da avaliação da resposta do GH (imunofluorimétrico) pós-estímulo e ressonância nuclear magnética (RNM) da região hipotálamo-hipofisária.

Nos pacientes que apresentavam puberdade precoce ou tardia, foram realizados: teste do GnRH para dosagens de LH e FSH (imunofluorimétrico) pós-estímulo, dosagens séricas de estradiol, progesterona e testosterona, além de ultrassonografia pélvica.

Nos pacientes com manifestações clínicas ou de risco para desenvolvimento de alterações da tireoide, foram solicitadas dosagens séricas de T3 total, T4 livre, TSH (fluoroimunoensaio), além dos anticorpos antitireoidianos (imunofluorimétrico). De acordo com os dados clínicos e laboratoriais, a investigação foi ampliada com ultrassonografia da tireoide e punção aspirativa por agulha fina (Paaf). 
Os pacientes também foram avaliados do ponto de vista metabólico, com dosagens de glicemia, insulinemia (imunofluorimétrico), colesterolemia (total e frações) e trigliceridemia e, quando necessário, submetidos a teste oral de tolerância à glicose (GTTo).

Considerou-se baixa estatura pacientes abaixo do percentil $5(\mathrm{z}<1,96)$ de estatura, puberdade precoce nas meninas a presença de eventos puberais (telarca e/ ou pubarca) antes dos 8 anos e a presença de eventos puberais antes dos 9 anos nos meninos; puberdade atrasada a ausência de brotos mamários em meninas e a falta de desenvolvimento testicular em meninos a partir de 14 anos; a obesidade foi caracterizada pelo IMC acima do $\mathrm{p}>95$ pela tabela do National Center for Health Statistics (NCHS) e dislipidemia índices baseados nos critérios de Stephen e cols. (14).

A análise estatística foi realizada pelo programa GraphPad InStat, sendo utilizados teste do qui-quadrado e teste de Wilcoxon (dados pareados) e Mann-Whitney (dados não pareados). Nível de significância foi considerado para $\mathrm{p}<0,05$.

O trabalho recebeu a ciência da Comissão de Ética para Análise de Projetos de Pesquisa (CAPPesq) do Hospital das Clínicas da FMUSP.

\section{RESULTADOS}

Dos pacientes que apresentaram alterações endócrinas, $52(55 \%)$ eram do sexo feminino.

A média de idade ao diagnóstico oncológico foi de 3 anos, sendo a mediana de 4,4 ( 0,1 a 14,7 anos).
A média de idade da primeira avaliação endocrinológica chegou a 12,1 anos e a mediana de 11,6 (2 a 27 anos). $\mathrm{O}$ intervalo médio entre o diagnóstico e a primeira avaliação endocrinológica foi de 7,4 anos, com mediana de 7,6 (0,5 a 21,4 anos).

$\mathrm{Na}$ tabela 1 , são apresentados as neoplasias acompanhadas pelo ambulatório de Fora de Terapia, os dados de pacientes submetidos à radioterapia e os que apresentaram efeitos endócrinos.

Constatou-se associação entre a realização de radioterapia e a presença de efeitos endócrinos $(\mathrm{X} 2=21,69$ p: 0,000003).

\section{Efeitos endócrinos tardios}

A tabela 2 apresenta os efeitos endócrinos encontrados nas várias neoplasias e sua frequência.

Dos noventa e quatro pacientes, noventa e sete efeitos endócrinos tardios foram observados: baixa estatura, puberdade precoce, tireoidopatias, obesidade, diabetes insípido, pan-hipopituitarismo e puberdade atrasada.

Trinta e dois pacientes evoluíram com baixa estatura durante o seguimento; destes, $20(64,5 \%)$ foram submetidos à radioterapia previamente. Dos 32 pacientes com baixa estatura, a deficiência de hormônio de crescimento $(\mathrm{GH})$ foi diagnosticada em nove, tendo recebido terapêutica de substituição. Um apresentou nódulo hepático em fígado transplantado durante a reposição hormonal, tendo sido optado pela suspensão da reposição do $\mathrm{GH}$, dois estavam em uso simultâneo de análogo do GnRH e os outros 23 continuaram em seguimento e/ou investigação diagnóstica.

Tabela 1. Frequência de neoplasias - proporção entre pacientes irradiados e com efeitos endócrinos

\begin{tabular}{|c|c|c|c|c|c|c|c|c|c|}
\hline \multirow{2}{*}{ Tabela de neoplasias } & & \multicolumn{2}{|c|}{ RDT } & \multicolumn{2}{|c|}{$\mathrm{EE}$} & \multirow{2}{*}{$\begin{array}{c}\text { RDT SIM } \\
+ \\
\text { EE SIM }\end{array}$} & \multirow{2}{*}{$\begin{array}{c}\text { RDT SIM } \\
+ \\
\text { EE Não }\end{array}$} & \multirow{2}{*}{$\begin{array}{c}\text { RDT NÃO } \\
+ \\
\text { EE SIM }\end{array}$} & \multirow{2}{*}{$\begin{array}{c}\text { RDT NÃO } \\
+ \\
\text { EE NÃO }\end{array}$} \\
\hline & & Sim & Não & Sim & Não & & & & \\
\hline Hepatoblastoma & 4 & 0 & 4 & 2 & 2 & 0 & 0 & 2 & 2 \\
\hline Histiocitose X & 11 & 3 & 8 & 9 & 4 & 2 & 0 & 5 & 4 \\
\hline Leucemias & 121 & 75 & 46 & 36 & 86 & 34 & 42 & 9 & 36 \\
\hline Linfomas & 60 & 41 & 19 & 15 & 45 & 11 & 28 & 3 & 18 \\
\hline Neuroblastoma & 24 & 1 & 23 & 1 & 23 & 0 & 1 & 1 & 22 \\
\hline Outros & 22 & 5 & 17 & 4 & 18 & 2 & 2 & 1 & 17 \\
\hline Retinoblastoma & 15 & 6 & 9 & 1 & 14 & 2 & 4 & 1 & 8 \\
\hline Sarcoma de Ewing & 12 & 10 & 2 & 1 & 11 & 1 & 10 & 0 & 1 \\
\hline Tumores do SNC & 23 & 21 & 2 & 22 & 1 & 15 & 7 & 0 & 1 \\
\hline Tumores de Wilms & 28 & 8 & 20 & 6 & 22 & 1 & 7 & 3 & 17 \\
\hline Total & 320 & 170 & 150 & 97 & 226 & $68^{a}$ & $101^{a}$ & $25^{a}$ & $126^{a}$ \\
\hline
\end{tabular}

RDT: radioterapia; EE: efeitos endócrinos; SNC: sistema nervoso central.

a - p: 0,001 . 
Tabela 2. Tipos de neoplasias, idades do início e da primeira consulta endocrinológica, tempo entre idade e primeira consulta, radioterapia e efeitos endócrinos

\begin{tabular}{|c|c|c|c|c|c|c|c|c|c|}
\hline \multirow[t]{2}{*}{$\begin{array}{l}\text { Tipos de } \\
\text { neoplasias } \\
\text { (n) }\end{array}$} & \multirow{2}{*}{$\begin{array}{c}\begin{array}{c}\text { Idade do } \\
\text { início da doença } \\
\text { (anos) }\end{array} \\
\text { Média (DP) }\end{array}$} & \multirow{2}{*}{$\begin{array}{c}\begin{array}{c}\text { Idade da } \\
\text { primeira consulta } \\
\text { endócrino (anos) }\end{array} \\
\text { Média (DP) } \\
\end{array}$} & \multirow{2}{*}{$\begin{array}{c}\text { Dif. } \\
\begin{array}{c}\text { Idade da doença } \\
\text { primeira consulta } \\
\text { endócrino (anos) }\end{array} \\
\text { Média (DP) }\end{array}$} & \multicolumn{2}{|c|}{ RDT } & \multirow[t]{2}{*}{$\begin{array}{l}\text { Alterações } \\
\text { endócrinas } \\
\text { (n) }\end{array}$} & & \multicolumn{2}{|c|}{ RDT } \\
\hline & & & & Sim & Não & & & Sim & Não \\
\hline \multirow{6}{*}{$\begin{array}{l}\text { Leucemias (35) } \\
\qquad \begin{array}{l}\text { LLA (29) } \\
\text { LMA (5) } \\
\text { LMC (1) }\end{array}\end{array}$} & $2,9(2,7)$ & $11,9(4,2)$ & $6,6(5,7)$ & 20 & 15 & Baixa estatura & 16 & 11 & 5 \\
\hline & & & & & & Puberdade precoce & 9 & 6 & 3 \\
\hline & & & & & & Obesidade & 7 & 2 & 4 \\
\hline & & & & & & Hipotireoidismo & 2 & 0 & 1 \\
\hline & & & & & & Tireoidite linfocitária crônica & 1 & 1 & 1 \\
\hline & & & & & & Diabetes melito & 1 & 0 & 1 \\
\hline \multirow{6}{*}{$\begin{array}{l}\text { Tumores do SNC (19) } \\
\text { Meduloblastoma (9) } \\
\text { Glioma óptico (4) } \\
\text { Disgerminoma (2) } \\
\text { Astrocitoma (2) } \\
\text { Craniofaringeoma (1) } \\
\text { Ependimoma (1) }\end{array}$} & $4,5(3,1)$ & $10,5(6,7)$ & $5,9(3,6)$ & 18 & 1 & Baixa estatura & 11 & 11 & 0 \\
\hline & & & & & & Puberdade atrasada & 2 & 2 & 0 \\
\hline & & & & & & Pan-hipopituitarismo & 3 & 2 & 1 \\
\hline & & & & & & Puberdade precoce & 3 & 3 & 0 \\
\hline & & & & & & Obesidade & 2 & 2 & 0 \\
\hline & & & & & & Nódulo da tireoide & 1 & 1 & 0 \\
\hline \multirow{4}{*}{$\begin{array}{l}\text { Linfomas (15) } \\
\text { Doença de Hodgkin (12) } \\
\text { Linfoma não Hodgkin (3) }\end{array}$} & $5,9(2,2)$ & $14(4,1)$ & $8,8(4,5)$ & 11 & 4 & Baixa estatura & 1 & 0 & 1 \\
\hline & & & & & & Nódulo da tireoide & 3 & 2 & 1 \\
\hline & & & & & & Hipotireoidismo & 8 & 6 & 2 \\
\hline & & & & & & Obesidade & 3 & 2 & 1 \\
\hline \multirow[t]{4}{*}{ Histiocitose X (7) } & $3,8(3,2)$ & $13,2(6,1)$ & $9,6(7,9)$ & 2 & 4 & Diabetes insípido & 6 & 1 & 5 \\
\hline & & & & & & Obesidade & 1 & 0 & 2 \\
\hline & & & & & & Puberdade atrasada & 1 & 1 & 0 \\
\hline & & & & & & Puberdade precoce & 1 & 0 & 1 \\
\hline \multirow[t]{3}{*}{ Tumor de Wilms (6) } & $3,1(0,9)$ & $8,7(4,8)$ & $6,1(4,5)$ & 0 & 6 & Baixa estatura & 2 & 0 & 2 \\
\hline & & & & & & Nódulo da tireoide & 1 & 0 & 1 \\
\hline & & & & & & Obesidade & 3 & 0 & 2 \\
\hline \multirow{6}{*}{$\begin{array}{l}\text { Menos frequentes (9) } \\
\text { Hepatoblastoma (2) } \\
\text { Rabdomiossarcoma (2) } \\
\text { Tumor de nasofaringe (2) } \\
\text { Retinoblastoma (1) } \\
\text { Neuroblastoma (1) } \\
\text { Sarcoma de Ewing (1) }\end{array}$} & $5,5(4,7)$ & $13,6(4,4)$ & $9,7(3,9)$ & 6 & 2 & Baixa estatura & 2 & 1 & 1 \\
\hline & & & & & & Obesidade & 2 & 1 & 1 \\
\hline & & & & & & Puberdade atrasada & 1 & 1 & 0 \\
\hline & & & & & & Hipotireoidismo & 2 & 2 & 0 \\
\hline & & & & & & Nódulo da tireoide & 1 & 0 & 1 \\
\hline & & & & & & & & & \\
\hline
\end{tabular}

LLA: leucemia linfocítica aguda, LMA: leucemia mieloide aguda; LMC: leucemia mieloide crônica; SNC: sistema nervoso central; RDT: radioterapia; DP: desvio-padrão; n: número de pacientes.

Com relação aos nove que receberam hormônio de crescimento recombinante humano $(\mathrm{rHGH})$, os pacientes iniciaram o tratamento aos 12,6 anos (média), sendo tratados por um período de 2,7 anos. O z inicial de estatura foi - 3 (1) e o $\mathbf{z}$ após o tempo de tratamento foi $-3,2(0,9)(p>0,05)$.

Catorze pacientes $(10 \mathrm{~F})$ apresentaram puberdade precoce. A idade da primeira consulta foi $9 \pm 1,8$ anos. A idade ao diagnóstico da doença de base foi 3,3 $\pm 2,6$ anos e a diferença entre o diagnóstico da doença de base e a primeira consulta foi $5,7 \pm 2,9$ anos.
Desses catorze pacientes, dez receberam análogo de $\mathrm{GnRH}$. Sete haviam sido submetidos à radioterapia. A idade de início de tratamento foi 9,6 $\pm 1,9$ anos. $\mathrm{O} Z$-score de estatura inicial foi $-0,5(-4,6$ a 0,6$)$. O tempo de tratamento foi $1,1 \pm 0,8$ ano. O $Z$-score de estatura após o período de observação foi $-0,7(-0,3$ a $-3,8)$. Não houve diferença significante na variação do $\mathbf{z}$ de estatura no período observado ( $\mathrm{p}>0,05)$.

Dos outros quatro pacientes que não foram tratados, um paciente do sexo masculino iniciou quadro de puberdade precoce em virtude de um tumor de células 
germinativas e chegou para avaliação aos 11 anos e 6 meses com altura de $154 \mathrm{~cm}$ e idade óssea de 16 anos. Duas outras pacientes foram tratadas por LLA com radioterapia e apresentaram menarca aos 9 e 10 anos, respectivamente. $\mathrm{O}$ quarto paciente, do sexo masculino, tratado de LLA, apresentou puberdade, que se iniciou aos 8 anos e 6 meses e evoluiu com desenvolvimento puberal adequado, estando em seguimento clínico.

Dezoito pacientes foram encaminhados para avaliação de obesidade, dos quais sete apresentaram níveis de colesterol total e/ou triglicérides elevados.

Eles foram iniciados em programa de reorientação alimentar na idade de $12,4 \pm 5,5$ anos por um período médio de 1,8 $\pm 0,9$ ano. Dois adolescentes receberam sibutramina $10 \mathrm{mg} /$ dia como parte de tratamento.

Não houve diferença significante nos valores de IMC, colesterol total, triglicérides, glicemia, insulina, relação glicemia/insulina antes e após o período de observação $(\mathrm{p}>0,05)$.

Em 19 pacientes manifestaram-se alterações da tireoide, sendo 12 com hipotireoidismo, seis com nódulos da tireoide e um com tireoidite linfocitária crônica.

Nos pacientes com nódulos da tireoide, quatro tinham cistos coloides estando em seguimento, um possuía bócio adenomatoso e uma paciente, cuja doença de base foi um hepatoblastoma, não tendo sido submetida à radioterapia local ou sistêmica, evoluiu com carcinoma papilífero da tireoide 13 anos após o aparecimento da neoplasia de base, sendo submetida à tireoidectomia total.

Seis dos doze pacientes com hipotireoidismo foram submetidos à radioterapia. $\mathrm{O}$ tempo médio de manifestação da moléstia foi 8,4 anos após o diagnóstico da neoplasia de base. Seis pacientes estão em reposição hormonal e os outros seis, em seguimento. O único com tireoidite linfocitária crônica apresentou LLA, não tendo sido submetido à radioterapia, estando em reposição hormonal.

Os pacientes com diabetes insípido (6) receberam reposição hormonal com DDAVP e os pacientes com puberdade atrasada receberam reposição hormonal apropriada ao sexo.

\section{DISCUSSÃO}

A taxa de sobrevida dos pacientes submetidos à terapêutica oncológica apresentou uma importante melhora nos últimos anos. O desenvolvimento de métodos mais precisos para o diagnóstico e a padronização de protocolos para tratamento contribuíram considera- velmente para o aumento da sua expectativa de vida. Como consequência, complicações decorrentes do tratamento passaram a ocorrer de maneira mais significativa, particularmente na faixa etária pediátrica $(1,3)$.

Uma das alterações endócrinas mais frequentes é baixa estatura, inclusive nos pacientes submetidos à irradiação craniana e/ou espinhal $(3,17)$.

Silva Alves e cols. (4) mostraram que pacientes submetidos a tratamento radioterápico associado à corticoterapia apresentam maior déficit de altura final quando comparados a pacientes que apenas receberam glicocorticoides, porém não conseguiram demonstrar correlação com a dose de irradiação nos 20 pacientes estudados (4).

Segundo Browstein e cols. (5), cinco variáveis correlacionaram-se de maneira independente com um aumento no $Z$-score do desvio-padrão de altura dos pacientes sobreviventes de câncer. São elas: uso de baixas doses de radiação na coluna vertebral, uso de altas doses de reposição de $\mathrm{GH}$, início do $\mathrm{GH}$, sexo masculino e ausência de outras endocrinopatias associadas (5).

Sklar (1) demonstrou maior agravo na estatura de pacientes jovens que receberam doses superiores a 30 Gy de irradiação no crânio e/ou na coluna vertebral (15). Couto-Silva e cols. (7) revelaram dados da literatura correlacionando doses de radiação com deficiência de $\mathrm{GH}$, a qual ocorreu em $56 \%$ dos pacientes submetidos a 24 Gy e em 100\% dos irradiados com doses superiores a $45 \mathrm{~Gy}$.

A taxa de baixa estatura encontrada nos pacientes aqui estudados atingiu $32,8 \%$, dos quais $67 \%$ foram submetidos à radioterapia.

Embora ainda seja controverso, o consenso da Sociedade de Endocrinologia Pediátrica Lawson-Wilkins aprovou o uso de GH em sobreviventes de câncer (16), recomendando-se iniciar o tratamento por um período mínimo de dois anos, fundamentalmente em tumores do sistema nervoso central (SNC), como meduloblastoma e craniofaringeomas (17). O número de pacientes que receberam hormônio de crescimento recombinante humano (rhGH) em nossa casuística ainda é pequeno, mas a idade de início de tratamento (12,6 anos) é um pouco tardia, podendo levar a prejuízo na altura final desses pacientes.

Alterações no desenvolvimento puberal (puberdade precoce ou atrasada) também são descritas na literatura, dependendo do tipo de tratamento ao qual o paciente foi submetido $(1,3)$. Os do sexo feminino com LLA tratada com radioterapia craniana tendem a apresentar 
puberdade precoce, enquanto as irradiadas em abdome ou eixo cranioespinhal podem evoluir tanto com puberdade precoce quanto atrasada (4).

Os tumores de SNC e a irradiação craniana podem desencadear puberdade precoce em alguns pacientes $(3,4,6,8)$. Fatores como tipo e localização do tumor (gliomas ópticos, astrocitomas e hamartomas) e doses de irradiação podem influenciar no aparecimento desse quadro clínico (3).

Dados da literatura sugerem que o uso de agonistas do hormônio liberador de gonadotrofinas $(\mathrm{GnRH})$ pode melhorar a altura final desses pacientes, particularmente quando associado ao uso do hormônio de crescimento (5).

Catorze pacientes acompanhados no nosso serviço evoluíram com puberdade precoce e 10 haviam sido submetidos a tratamento radioterápico. $\mathrm{O}$ tratamento com análogo do $\mathrm{GnRH}$ foi feito em 10 deles por um período médio de um ano, os quais não apresentaram alteração significante.

Nos pacientes com doença de Hodgkin, em especial os que receberam irradiação cervical, a incidência de tireoidopatias aumenta consideravelmente, incluindo $\mathrm{o}$ aparecimento de nódulos e tumores $\mathrm{O}$ seguimento deve ser a longo prazo, uma vez que a evolução pode levar até 20 anos $(1,3,8-8,12,13)$.

O hipotireoidismo é o distúrbio que mais frequentemente acomete a glândula tireoide após esta ter sido submetida a algum grau de irradiação. Cerca de 30\% dos pacientes que sobrevivem à doença de Hodgkin desenvolvem hipotireoidismo e de $2 \%$ a $65 \%$ podem apresentar nódulos tireoidianos. Naqueles com história prévia de tumor de $\mathrm{SNC}$, a taxa de hipotireoidismo pode atingir $60 \%$, dependendo do tipo de tratamento ao qual eles foram submetidos $(1,3,7,8)$.

Em nossa casuística, dos 12 pacientes com hipotireoidismo, seis foram submetidos à radioterapia e o tempo entre a doença de base e este quadro foi de 8,4 anos. O hipotireoidismo foi mais prevalente nos que tiveram como doença de base linfomas, sendo que 12 dos 15 apresentaram linfoma de Hodgkin, dado esse que coincide com estudos anteriores (8).

Com relação ao câncer da tireoide, fatores com doses de radioterapia, tempo após o tratamento, são influentes no aparecimento dessa neoplasia $(12,13)$. Contudo, o tratamento quimioterápico prévio, nesse estudo, parece não se correlacionar com o desenvolvimento de nódulos de tireoide durante o seguimento clínico dos pacientes (13).
Dos seis pacientes com nódulos tireoideanos, três foram submetidos à radioterapia cervical para tratamento de linfoma de Hodgkin e um, à radioterapia craniana e eixo para tratamento de meduloblastoma. A diferença entre o aparecimento dos nódulos e da doença de base foi de 9,4 anos. Uma paciente apresentou carcinoma papilífero da tireoide aos 16,8 anos, 13,8 anos depois de ter sido submetida a tratamento para hepatoblastoma, cujo protocolo não incluía radioterapia.

A obesidade tem sido descrita como importante sequela tardia do tratamento oncológico, preponderantemente naqueles que apresentam antecedente de LLA e tumores de SNC. Nos pacientes com irradiação craniana, o aumento de peso foi dose dependente $(5,7,9,10,11)$.

A principal hipótese para o desenvolvimento da obesidade é que ocorra lesão nos centros hipotalâmicos de controle de saciedade. Outra razão para que os pacientes apresentem ganho excessivo de peso é uma alteração no "feedback" da leptina no hipotálamo, levando a um desequilíbrio no controle vagal de secreção de insulina, ocasionando um hiperinsulinismo nem sempre associado a aumento de resistência insulínica $(3,6,7,8)$.

Apenas alguns de nossos pacientes evoluíram com aumento de IMC após tratamento de neoplasia de SNC. Tal fato pode ser explicado pelo pequeno número de pacientes com esse tipo de tumor presente em nossa casuística. A maioria dos nossos pacientes obesos apresentou antecedente de LLA.

Com relação à idade de encaminhamento, eles eram inicialmente encaminhados após cinco anos de terapia, mas esse encaminhamento foi sendo reduzido, em princípio, para um período de dois anos passado o término da terapia, percebendo-se claramente a necessidade de encaminhamento e intervenção mais precoce dos efeitos endócrinos secundários, quando a evolução oncológica assim o permitisse.

Outros fatores que também merecem ser analisados são as medicações usadas no tratamento quimioterápico, embora isso não tenha feito parte deste estudo. Outras manifestações endócrinas, como puberdade atrasada e diabetes insípido, não foram aqui motivo de análise em virtude de sua baixa frequência.

Portanto, pacientes que foram submetidos a tratamento oncológico devem ser seguidos precoce e regularmente para possibilitar o diagnóstico de alterações endócrinas e propiciar-lhes, além de maior taxa de sobrevida, melhor qualidade de vida. 
Novos estudos devem ser incentivados com o firme propósito de elucidar os mecanismos fisiopatológicos pelos quais se estabelecem as sequelas endocrinológicas dos pacientes Fora de Terapia oncológica, para que intervenções e tratamentos mais precoces possam ser instituídos.

Declaração: os autores declaram não haver conflitos de interesse científico neste estudo.

\section{REFERÊNCIAS}

1. Sklar C. Endocrine complications of the sucessful treatment of neoplasic diseases in childhood. Growth Genet Horm. 2001;17(3):37-9.

2. Oeffinger KC, Mertens AC, Sklar CA, Kawashima T, Hudson MM Meadows AT, et al. Chronic health conditions in adult survivors of childhood cancer. N Engl J Med. 2006;355(15):1572-82.

3. Cohen LE. Endocrine late effects of cancer treatment. Endocrinol Metab Clin North Am. 2005;34(3):769-89.

4. Silva Alves CHB, Kuperman H, Dichtchekenian V, Damiani D, Della MannaT, Cristofani LM, et al. Growth and puberty after treatment for acute lymphoblastic leukemia. Rev Hosp Clin Fac Med S Paulo. 2004;59(2):67-70.

5. Browstein CM, Mertens AC, Mitby PA, Stovall M, Qin J, Heller G, et al. Factors that affect final height and change in height standard deviation scores in survivors of childhood cancer treated with growth hormone: a report from the childhood cancer survivor study. J Clin Endocrinol Metab. 2004;89(9):4422-7.

6. Rose SR. Endocrinopathies in childhood cancer survivors. Endocrinologist. 2003;13(6):488-95.

7. Couto-Silva AC, Brauner R, Adan LF. Seqüelas endócrinas da radioterapia no tratamento do câncer na infância e adolescência. Arq Bras Endocrinol Metab. 2005;49(5):825-32.
8. Gleeson HK, Shalet SM. The impact of cancer therapy on the endocrine system in survivors of childhood brain tumours. Endocr Relat Cancer. 2004;11(4):589-602.

9. Sklar CA, Mertens AC, Walter A, Mitchell D, Nesbit ME, O'Leary M, et al. Changes in body mass index and prevalence of overweight in survivors of childhood acute lymphoblastic leukemia: role of cranial irradiation. Med Pediatr Oncol. 2000;35(2):91-5.

10. Oeffinger KC, Mertens AC, Sklar, CA, Yasui Y, FearsT, Stovall M, et al. Obesity in adult survivors of childhood acute lymphoblastic leukemia: a report from the childhood cancer survivors study. $J$ Clin Oncol. 2003;21(7):1359-65.

11. Warner JT, Evans WD, Webb DKH, Gregory JW. Body composition of long-term survivors of acute lymphoblastic leukemia. Med Pediatr Oncol. 2002;38(3):165-72.

12. Acharya S, Sarafoglou K, LaQuaglia M, Lindsley S, Gerald W, Wollner $\mathrm{N}$, et al. Thyroid neoplasms after therapeutic radiation for malignancies during childhood or adolescence. Cancer. 2003;97(10):2397-403.

13. Sigurdson A, Ronckers CM, Mertens AC, Stovall M, Smith SA, Liu $Y$, et al. Primary thyroid cancer after a first tumor in childhood (the childhood cancer survivor study): a nested case-control study. Lancet. 2005;365(9476):2014-23.

14. Daniels SR, Greer FR; Committee on Nutrition. Lipid screening and cardiovascular health in childhood. Pediatrics 2008;122;198-208.

15. Chemaitilly W, Boulad F, Heller G, Kernan NA, Small TN, O'Reilly RJ, et al. Final height in pediatric patients after hyperfractionated total body irradiation and stem cell transplantation. Bone Marrow Transplant. 2007;40(1):29-35.

16. Sperling MA, Saenger PH, Ray Hintz, Tom Wilson, Rose SR; Lawson Wilkins Pediatric Endocrine Society. LWPES Executive Committee and the LWPES Drug andTherapeutics Committee. Growth hormone treatment and neoplasia - coincidence or consequence? J Clin Endocrinol Metab. 2002;87(12):5351-2.

17. Swerdlow AJ, Reddingius RE, Higgins CD, Spoudeas HA, Phipps $K$, Qiao Z, et al. Growth hormone treatment of children with brain tumors and risk of tumor recurrence. J Clin Endocrinol Metab. 2000;85(12):4444-9. 